\title{
PROBABILISTIC WELL-POSEDNESS FOR SUPERCRITICAL WAVE EQUATIONS WITH PERIODIC BOUNDARY CONDITION ON DIMENSION THREE
}

\author{
CHENMIN SUN AND BO XIA
}

\begin{abstract}
In this article, by following the strategies in dealing with supercritical cubic and quintic wave equations in (J. Eur. Math. Soc. (JEMS) 16 (2014) 1-30) and (J. Math. Pures Appl. (9) 105 (2016) 342-366), we obtain that, the equation

$$
\left(\partial_{t}^{2}-\Delta\right) u+|u|^{p-1} u=0, \quad 3<p<5
$$

is almost surely global well-posed with initial data $\left(u(0), \partial_{t} u(0)\right) \in$ $H^{s}\left(\mathbb{T}^{3}\right) \times H^{s-1}\left(\mathbb{T}^{3}\right)$ for any $s \in\left(\frac{p-3}{p-1}, 1\right)$. The key point here is that $\frac{p-3}{p-1}$ is much smaller than the critical index $\frac{3}{2}-\frac{2}{p-1}$ for $3<p<5$.
\end{abstract}

\section{Introduction}

In this article, we are going to construct solutions for the equation

$$
\left\{\begin{array}{l}
\left(\partial_{t}^{2}-\Delta\right) u+|u|^{p-1} u=0, \quad 3<p<5 \\
\left.\left(u, \partial_{t} u\right)\right|_{t=0}=\left(u_{0}, u_{1}\right) \in H^{s} \times H^{s-1}=: \mathcal{H}^{s}
\end{array}\right.
$$

where $u$ is a real-valued function defined on $\mathbb{R}_{t} \times \mathbb{T}^{3}$. Via a scaling argument, one can see that $s_{\mathrm{cr}}=\frac{3}{2}-\frac{2}{p-1}$ is a critical index in solving the equation (1.1). It turns out that for $s<s_{\mathrm{cr}}$, the equation (1.1) is ill-posed, while for $s \geq s_{\mathrm{cr}}$, the equation (1.1) is known to be well-posed (in the sense of Hadamard) only for $s$ in certain range. More precisely, we have the following theorem.

THEOREM 1.1. The Cauchy problem (1.1) is locally well-posed for data in $\mathcal{H}^{s}$ for $s>s_{\mathrm{cr}}$. In the opposite direction, for $p \in[3,5)$, if $s \in\left(0, \frac{3}{2}-\frac{2}{p-1}\right)$, then

Received August 3, 2015; received in final form March 1, 2017.

The first author is supported by Master program by University of Paris-Saclay, while the second author is supported by CSC.

2010 Mathematics Subject Classification. 35L05, 42B37. 
the problem (1.1) is not locally well-posed in $\mathcal{H}^{s}$. One example contradicting the continuous dependence on the initial data is as follows: there exists a sequence $\left(u_{n}\right)$ of global smooth solutions to (1.1) with initial data $\left(u_{0}^{(n)}, u_{1}^{(n)}\right)$ such that

$$
\lim _{n \rightarrow \infty}\left\|\left(u_{0}^{(n)}, u_{1}^{(n)}\right)\right\|_{\mathcal{H}^{s}}=0
$$

but

$$
\lim _{n \rightarrow \infty}\left\|\left(u_{n}(t), \partial_{t} u_{n}(t)\right)\right\|_{L^{\infty}\left([0, T] ; \mathcal{H}^{s}\right)}=\infty, \quad \forall T>0 .
$$

The well-posedness part of Theorem 1.1 can be proved in the same way as Lindblad-Sogge did in [13], by invoking the Strichartz estimate on compact manifold thanks to Kapitanski [10]. For the special case $p=3,(1.1)$ is even globally well-posed if the regularity index $s$ is sufficiently close to 1 . One can also refer to [19] for these results on the Euclidean space $\mathbb{R}^{3}$. For the ill-posedness statement of Theorem 1.1, one can see Burq-Tzvetkov [6, Appendix A] for $p=3$ and [20] for $3<p<5$, or see [9] for even more discussions.

Up to these counter examples, we can not solve the equation (1.1) in the sense of Hadamard in the super-critical regime. In order to construct solutions to these super-critical equations, probabilistic tools have been introduced. On one hand, for some special initial data, one can use an "invariant measure argument" to construct local or even global solutions to several equations in super-critical regime (see [3], [2], [12], [7] for detailed discussions). On the other hand, for general initial data, by randomizing the data via its Fourier series, Burq-Tzvetkov [6] succeeded in constructing local solutions to (1.1) in the super-critical regime. Shortly after this, they also proved the probabilistic global well-posedness of the cubic wave equation on 3D torus by a conservation law argument in [8]. Using this argument, Burq-Thomann-Tzvetkov obtained probabilistic global existence of solution to the cubic wave equation in higher dimensions in [5].

Recently Oh-Pocovnicu [17] proved the quintic wave equation on $\mathbb{R}^{3}$ is almost surely global well-posed with the initial data in the homogeneous space $\dot{\mathcal{H}}^{s}\left(\mathbb{R}^{3}\right):=\dot{H}^{s}\left(\mathbb{R}^{3}\right) \times \dot{H}^{s-1}\left(\mathbb{R}^{3}\right)$ with $s>\frac{1}{2}$. The approach Oh-Pocovnicu used is slightly different from Burq-Tzvetkov's. Here as the spectrum of Laplacian is continuous, we can not use the randomization procedure used by BurqTzvetkov to randomize data. Oh-Pocovnicu chose to use Wiener randomization $^{1}$ in [1], and they proved similar probabilistic estimates as in [8], [6]. Furthermore, they also proved probabilistic estimates for the time derivative of the free evolution of the data. By using these probabilistic estimates, the authors first establish a probabilistic a priori estimate. Then by combining

\footnotetext{
1 This randomization was first introduced by Zhang-Fang [21] for generalized incompressible Navier-Stokes systems. Subsequently, such randomization appeared in [14], which is part of thesis of both Lührmann and Mendelson. At last, this randomization was named as "Wiener randomization" in [1].
} 
this a priori estimate with the stability theory for NLW, they succeeded in constructing a global solution to quintic wave equation in $\dot{\mathcal{H}}^{s}$ with $s>\frac{1}{2}$.

Also on the Euclidean space $\mathbb{R}^{3}$, by randomizing the initial data via a unit-scale decomposition of the frequency space, Lührmann-Mendelson [14] proved similar probabilistic estimates as that in [6], [8]. With these estimates in hand, they proved that the problem (1.1) with the underground space replaced by $\mathbb{R}^{3}$ is almost surely global well-posed in $\dot{\mathcal{H}}^{s}$ for $s>\frac{p^{3}+5 p^{2}-11 p-3}{9 p^{2}-6 p-3}$. Notice that this regularity index only lies in the super-critical regime when $\frac{1}{4}(7+\sqrt{73})<p<5$. To remedy this, they improved in [15] this result to $1>s>\frac{p-1}{p+1}$ by using Oh-Pocovnicu's ideas in [17].

One should observe that, no matter the underlying space is the torus $\mathbb{T}^{3}$ or the whole Euclidean space $\mathbb{R}^{3}$, we have almost the same probabilistic estimates. So we shall expect that the best possible regularity $s(p)$ required to solve (1.1) should be compatible with the two endpoint cases $p=3$ and $p=5$, in the sense that

$$
s(p) \rightarrow_{p \rightarrow 3} 0=s(3) \text { and } \quad s(p) \rightarrow_{p \rightarrow 5} \frac{1}{2}=s(5),
$$

where $s(3)=0$ is the result by Burq-Tzvetkov [8] and $s(5)=\frac{1}{2}$ is the result by Oh-Pocovnicu [17]. However, Lührman-Mendelson's result $s(p)=\frac{p-1}{p+1}$ fails to behave like this. In this article, we are going to address this problem. Our approach is as follows, which is also the approach in [17].

Fix $s \in\left(\frac{p-3}{p-1}, 1\right)$. Let $\left(u_{0}, u_{1}\right) \in \mathcal{H}^{s}$ be given in its Fourier series form

$$
u_{j}=a_{j, 0}+\sum_{n \in \mathbb{Z}_{+}^{3}}\left(a_{j, n} \cos (n \cdot x)+b_{j, n} \sin (n \cdot x)\right), \quad j=0,1,
$$

where $\mathbb{Z}_{+}^{3}:=\left\{\left(n_{1}, n_{2}, n_{3}\right) \in \mathbb{Z}^{3} \mid n_{1} \geq 0\right\} \backslash\left(\left\{\left(0, n_{2}, n_{3}\right) \in \mathbb{Z}^{3} \mid n_{2}<0\right\} \cup\right.$ $\left.\left\{\left(0,0, n_{3}\right) \in \mathbb{Z}^{3} \mid n_{3} \leq 0\right\}\right)$. Suppose $\left(\alpha_{j}(\omega), \beta_{j, n}(\omega), \gamma_{j, n}(\omega)\right)_{j=0,1 ; n \in \mathbb{Z}_{+}^{3}}$ is a series of independent real Gaussians on $(\Omega, \mathcal{A}, \mathbb{P})$ with standard distribution $\mathcal{N}_{\mathbb{R}}(0,1)$, or even more general that they satisfy the assumption $(2.3)$. Then we define the random variables $u_{j}^{\omega}$ as

$u_{j}^{\omega}=a_{j, 0} \alpha_{j}(\omega)+\sum_{n \in \mathbb{Z}_{+}^{3}}\left(a_{j, n} \beta_{j, n}(\omega) \cos (n \cdot x)+b_{j, n} \gamma_{j, n}(\omega) \sin (n \cdot x)\right), \quad j=0,1$.

For any integer $N \geq 1$, denote by $P_{N}$ the projection operator defined by

$$
\begin{aligned}
P_{\leq N}\left(a_{0}+\sum_{n \in \mathbb{Z}_{+}^{3}}\left(a_{n} \cos (n \cdot x)+b_{n} \sin (n \cdot x)\right)\right) \\
=a_{0}+\sum_{n \in \mathbb{Z}_{+}^{3} ;|n| \leq N}\left(a_{n} \cos (n \cdot x)+b_{n} \sin (n \cdot x)\right) .
\end{aligned}
$$


Denote also the free wave propagator by $S(t)$. Then we first prove that for any given $N \geq 1$ and $T, \varepsilon>0$, there exists a subset $\Omega_{N, T, \varepsilon} \subset \Omega$ with $\mathbb{P}\left(\Omega_{N, T, \varepsilon}^{c}\right)<\varepsilon$, the solution $v_{N}$ to the truncated equation

$$
\left\{\begin{array}{l}
\left(\partial_{t}^{2}-\Delta\right) v_{N}+\left|v_{N}+z_{N}\right|^{p-1}\left(v_{N}+z_{N}\right)=0, \quad z_{N}=S(t)\left(P_{\leq N} u_{0}^{\omega}, P_{\leq N} u_{1}^{\omega}\right), \\
\left.\left(v_{N}, \partial_{t} v_{N}\right)\right|_{t=0}=(0,0)
\end{array}\right.
$$

can be bounded uniformly as

$$
\sup _{t \in[0, T]}\left\|\left(v_{N}(t), \partial_{t} v_{N}(t)\right)\right\|_{\mathcal{H}^{1}} \leq C,
$$

where $C$ depends only on $T, \varepsilon$ and $\left\|\left(u_{0}, u_{1}\right)\right\|_{\mathcal{H}^{s}}$, and is independent of $N$. The main step in the proof of this estimate is Lemma 3.3, in which we have used an adapted cut-off argument.

With the help of (1.2), we could find another set $\tilde{\Omega}_{N, T, \varepsilon}$, which may be different from $\Omega_{N, T, \varepsilon}$ and is also of large probability, such that for any $\omega \in \tilde{\Omega}_{N, T, \varepsilon}$, the solution $v(t)$ to the equation

$$
\left\{\begin{array}{l}
\partial_{t}^{2} v-\Delta v+|v+z|^{p-1}(v+z)=0, \quad z=S(t)\left(u_{0}^{\omega}, u_{1}^{\omega}\right), \\
\left.\left(v, \partial_{t} v\right)\right|_{t=0}=(0,0)
\end{array}\right.
$$

can also be controlled uniformly

$$
\sup _{t \in[0, T]}\left\|\left(v(t), \partial_{t} v(t)\right)\right\|_{\mathcal{H}^{1}}<2 C
$$

By a standard argument in [8], we can extend this finite time $[0, T]$ to the whole time line. And at last we arrive at the following theorem.

TheOREM 1.2 (Almost sure global well-posedness). Let $s \in\left(\frac{p-3}{p-1}, 1\right)$. Given $\left(u_{0}, u_{1}\right) \in \mathcal{H}^{s}\left(\mathbb{T}^{3}\right)$, let $\left(u_{0}^{\omega}, u_{1}^{\omega}\right)$ be the randomization as in $(2.5)$ under the assumption (2.3). Then the super-critical wave equation (1.1) is almost surely globally well-posed with $\left(u_{0}^{\omega}, u_{1}^{\omega}\right)$ as the initial data. More precisely, there exists a set $\Omega_{\left(u_{0}, u_{1}\right)} \subset \Omega$ of probability 1 such that, for every $\omega \in \Omega_{\left(u_{0}, u_{1}\right)}$, there exists a unique solution $u$ (in a bounded ball around zero) to (1.1) in the class:

$$
\left(S(t)\left(u_{0}^{\omega}, u_{1}^{\omega}\right), \partial_{t} S(t)\left(u_{0}^{\omega}, u_{1}^{\omega}\right)\right)+C\left(\mathbb{R} ; \mathcal{H}^{1}\left(\mathbb{T}^{3}\right)\right) \subset C\left(\mathbb{R} ; \mathcal{H}^{s}\left(\mathbb{T}^{3}\right)\right) .
$$

REMARK 1.3. This remark is corresponding to the title 'well-posedness': the uniqueness of the solution in the statement is in the sense of Remark 1.6(i) in [17]. But the (conditional) continuous dependence on the initial data should be explained in detail: for any given time $T>0$, one should notice that the solution $v$ constructed in Theorem 1.2 can be written as $v(t)=v_{N}(t)+S(t)\left(u_{0}^{\omega}, u_{1}^{\omega}\right)-z_{N}$ for any $t \in[0, T]$ for some very big but finite $N$; then by the deterministic theory in the energy space for the equation (1.1), $v_{N}(t)$ depends continuously on $\left(v_{N}(0), \partial_{t} v_{N}(0)\right)$, which turns out to be controlled by $\left(u_{0}^{\omega}, u_{1}^{\omega}\right)$ thanks to the boundedness of $N$ (at least in the sense of probability); this continuity, together with the fact that the linear evolution 
$S(t)\left(u_{0}^{\omega}, u_{1}^{\omega}\right)-z_{N}$ depends continuously on the initial data, can give us the conditional dependence on the initial data like that in [8]. What's more, one can also obtain the probabilistic growth for Sobolev norm of the solution via a detailed analysis as Burq-Tzvetkov did in [8].

REMARK 1.4. We should notice that the lower bound $\frac{p-3}{p-1}$ is compatible with the endpoint cases $p=3$ and $p=5$. That is to say, when $p$ tends to 3 , the minimal regularity required to solve the equation (1.1) becomes the one obtained in [8] for the case $p=3$; and the same for the other endpoint $p=5$, see [17]. But if $p=3$ and $s=0$, we refer to [8] for the possible growth of Sobolev norms.

REMARK 1.5. For higher dimensional case $d \geq 4$, the global infinite energy solution to the cubic wave equation was constructed by Burq-ThomannTzvetkov [5], where the conditionally continuous dependence on the initial data is left unknown. But Oh-Pocovnicu succeeded in proving this uniqueness result in [16].

\section{Preliminaries}

2.1. Deterministic preliminaries. In this section, we recall several classical results about the linear equation

$$
\left\{\begin{array}{l}
\left(\partial_{t}^{2}-\Delta\right) u=f \quad \text { on } I \times \mathbb{T}^{3} \\
\left.\left(u, \partial_{t} u\right)\right|_{t=t_{0}}=\left(u_{0}, u_{1}\right)
\end{array}\right.
$$

We say that $u$ solves the equation (2.1) on the time interval $I \ni t_{0}$ if $u$ satisfies for $t \in I$ the Duhamel formula

$$
u(t)=S\left(t-t_{0}\right)\left(u_{0}, u_{1}\right)+\int_{t_{0}}^{t} \frac{\sin \left(\left(t-t^{\prime}\right) \sqrt{-\Delta}\right)}{\sqrt{-\Delta}} f\left(t^{\prime}\right) d t^{\prime},
$$

where $S(t)$ is the free wave propagator defined by

$$
S(t)\left(u_{0}, u_{1}\right)=\cos (t \sqrt{-\Delta}) u_{0}+\frac{\sin (t \sqrt{-\Delta})}{\sqrt{-\Delta}} u_{1} .
$$

We now recall the following energy estimates for the solution $u$ to the equation (2.1).

Proposition 2.1 (Energy estimates). Suppose $u$ solves (2.1) on $I=$ $\left[t_{0}=0, T\right]$. Then for any $t \in[0, T]$ we have

$$
\left\|\left(u(t, \cdot), \partial_{t} u(t, \cdot)\right)\right\|_{\mathcal{H}^{s}} \leq C(1+T)\left(\left\|\left(u_{0}, u_{1}\right)\right\|_{\mathcal{H}^{s}}+\int_{0}^{t}\|f(r, \cdot)\|_{H^{s-1}} d r\right) .
$$

We also use frequently the following Strichartz estimate, which is very useful in dealing with the nonlinearity in the equation (1.1). In order to state this estimate, we first define the concept of "wave-admissibility" in 3D case. 
Definition 2.2. We call a pair $(q, r)$ wave-admissible if $2 \leq q \leq \infty$, $2 \leq r \leq \infty,(q, r) \neq(2, \infty)$ and

$$
\frac{1}{q}+\frac{1}{r} \leq \frac{1}{2}
$$

Proposition 2.3 (Strichartz estimates for wave equation [11], [10]). Let u be the solution to $(2.1)$ on any time interval $0 \in I \subset[0,1]$, we have

$$
\|u\|_{L^{p}\left(I ; L^{q}\left(\mathbb{T}^{3}\right)\right)} \leq C\left(\left\|\left(u_{0}, u_{1}\right)\right\|_{\mathcal{H}^{s}}+\|f\|_{L^{a^{\prime}}\left(I ; L^{b^{\prime}}\left(\mathbb{T}^{3}\right)\right)}\right)
$$

under the assumptions that

(1) wave admissible condition: both the pairs $(p, q)$ and $(a, b)$ are waveadmissible;

(2) scaling invariant condition:

$$
\frac{1}{p}+\frac{3}{q}=\frac{1}{a^{\prime}}+\frac{3}{b^{\prime}}-2=\frac{3}{2}-s .
$$

Indeed, in our case, the Strichartz type estimate we use is mainly for the pair $\left(\frac{2 p}{p-3}, 2 p\right)$ with regularity $s=1$ and the pair $(\infty, 2)$ with $s=0$. Precisely, what we need is the following estimate

$$
\left\|\left(u, \partial_{t} u\right)\right\|_{L_{t}^{\infty}\left(I ; \mathcal{H}_{x}^{1}\right)}+\|u\|_{L_{t}^{\frac{2 p}{p-3}}\left(I, L_{x}^{2 p}\right)} \leq\left\|\left(u_{0}, u_{1}\right)\right\|_{\mathcal{H}^{1}}+\|f\|_{L_{t}^{1}\left(I ; L_{x}^{2}\right)}
$$

for any time interval $I$ containing $t_{0}$ with $|I| \leq 1$. In the following, we denote $\phi_{0}$ a radial smooth function on $\mathbb{R}^{3}$ such that $\phi_{0}=1$ on the ball $B(0,1)$ and $\phi_{0}=0$ outside the ball $B(0,2)$. Then we recall the following projection operators for any integer $N \geq 1$

$$
P_{\leq N} u=a_{0}+\sum_{n \in \mathbb{Z}_{+}^{3}} \phi_{0}\left(\frac{|n|}{N}\right)\left(a_{n} \cos (n \cdot x)+b_{n} \sin (n \cdot x)\right)
$$

provided that $u$ is given by

$$
u=a_{0}+\sum_{n \in \mathbb{Z}_{+}^{3}} a_{n} \cos (n \cdot x)+b_{n} \sin (n \cdot x),
$$

where $\mathbb{Z}_{+}^{3}:=\left\{\left(n_{1}, n_{2}, n_{3}\right) \in \mathbb{Z}^{3} \mid n_{1} \geq 0\right\} \backslash\left(\left\{\left(0, n_{2}, n_{3}\right) \in \mathbb{Z}_{+}^{3} \mid n_{2}<0\right\} \cup\right.$ $\left.\left\{\left(0,0, n_{3}\right) \in \mathbb{Z}^{3} \mid n_{3} \leq 0\right\}\right)$. When $N=2^{j}$ is a dyadic for some $j \geq 0$, we also define the projection operators

$$
P_{j} u:=P_{\leq 2^{j}} u-P_{\leq 2^{j-1}} u,
$$

where we have used the convention that $P_{<^{-1}} u=0$. Then by the classical Littlewood-Paley theory, we have the following characterization of $\mathrm{H}^{s_{-}}$ Sobolev spaces

$$
\|u\|_{H^{s}}^{2} \sim \sum_{j \geq 0} 2^{2 j s}\left\|P_{j} u\right\|_{L^{2}}^{2}
$$


We also need Bernstein's inequality

$$
\left\|P_{\leq N} u\right\|_{L^{q}} \leq N^{\frac{3}{p}-\frac{3}{q}}\left\|P_{\leq N} u\right\|_{L^{p}}, \quad 1 \leq p \leq q \leq \infty .
$$

\subsection{Probabilistic preliminaries. Now let}

$$
\left(\alpha_{j}(\omega), \beta_{n, j}(\omega), \gamma_{n, j}(\omega)\right)_{n \in \mathbb{Z}_{+}^{3}, j=0,1}
$$

be a series of independent identically distributed real, mean zero, random variables on the probability space $(\Omega, \mathcal{A}, \mathbb{P})$ with the same distribution function $\theta$. Assume that there exists $c>0$ such that

$$
\forall \gamma \in \mathbb{R}, \quad \int_{-\infty}^{+\infty} e^{\gamma x} d \theta(x) \leq e^{c \gamma^{2}}
$$

Using such a series of random variables, we randomize the data $\left(u_{0}, u_{1}\right) \in \mathcal{H}^{s}$, given by their Fourier series with all coefficients real

$$
u_{j}(x)=a_{j}+\sum_{n \in \mathbb{Z}_{+}^{3}}\left(b_{n, j} \cos (n \cdot x)+c_{n, j} \sin (n \cdot x)\right), \quad j=0,1
$$

by setting

$$
u_{j}^{\omega}(x)=\alpha_{j}(\omega) a_{j}+\sum_{n \in \mathbb{Z}_{+}^{3}}\left(\beta_{n, j}(\omega) b_{n, j} \cos (n \cdot x)+\gamma_{n, j}(\omega) c_{n, j} \sin (n \cdot x)\right) .
$$

REMARK 2.4. Indeed the map $\omega \longmapsto\left(u_{0}^{\omega}, u_{1}^{\omega}\right)$ induces a Borel probability measure on $\mathcal{H}^{s}$ equipped with its natural topology. Furthermore, this probability measure on $\mathcal{H}^{s}$ has many nice properties such as "non-regularization of the data" and "non-vanishing on any open set", which exclude the possibility of "regularizing effect" originating from such procedure when applied to PDE. See [8], [6] for more details.

We first recall the following estimates of higher moments of random series associated to any given $\ell^{2}$ sequence $\left(c_{n}\right)$, which is very important in obtaining probabilistic estimates for the random variables $\left(u_{0}^{\omega}, u_{1}^{\omega}\right)$.

LEMMA $2.5([6])$. Let $\left\{g_{n}\right\}$ be a sequence of independent mean-zero, realvalued random variables and the distribution function of $g_{n}$ satisfies the assumption (2.3) for any integer $n$. Then for any $\ell^{2}$ sequence $\left(c_{n}\right)$ and any $q \geq 2$, there exists $c>0$ such that

$$
\left\|\sum g_{n}(\omega) c_{n}\right\|_{L_{\omega}^{q}} \leq c \sqrt{q}\left\|\left(c_{n}\right)\right\|_{\ell^{2}} .
$$

By using this estimate, we can prove the following local-in-time probabilistic Strichartz estimates by using the ideas used in [6], [7], [18].

LEMMA $2.6([6],[7],[18])$. Let $\left(u_{0}, u_{1}\right) \in \mathcal{H}^{s}\left(\mathbb{T}^{3}\right)$ be given by the series (2.4) with all coefficients real and $\left(u_{0}^{\omega}, u_{1}^{\omega}\right)$ be randomized as in (2.5). Assume $I=[a, b] \subset \mathbb{R}$ is a compact time interval. 
(i) If $s=0$, then for any given $1 \leq q<\infty$ and $2 \leq r<\infty$, there exist $C, c>0$ such that

$$
\mathbb{P}\left(\left\|S(t)\left(u_{0}^{\omega}, u_{1}^{\omega}\right)\right\|_{L_{t}^{q} L_{x}^{r}\left(I \times \mathbb{T}^{3}\right)}>\lambda\right) \leq C \exp \left(-c \frac{\lambda^{2}}{|I|^{\frac{2}{q}}\left\|\left(u_{0}, u_{1}\right)\right\|_{\mathcal{H}^{0}}^{2}}\right) .
$$

(ii) For any given $1 \leq q<\infty, 2 \leq r \leq \infty$, there exist $C, c>0$ such that

$$
\begin{aligned}
& \mathbb{P}\left(\left\|S(t)\left(u_{0}^{\omega}, u_{1}^{\omega}\right)\right\|_{L_{t}^{q} L_{x}^{r}\left(I \times \mathbb{T}^{3}\right)}>\lambda\right) \leq C \exp \left(-c \frac{\lambda^{2}}{|I|^{\frac{2}{q}}\left\|\left(u_{0}, u_{1}\right)\right\|_{\mathcal{H}^{s}}^{2}}\right) \\
& \text { for (ii.a) } s=0 \text { if } r<\infty \text { and (ii.b) } s>0 \text { if } r=\infty .
\end{aligned}
$$

By denoting $\tilde{S}(t)$ by

$$
\tilde{S}(t)\left(u_{0}, u_{1}\right):=-\frac{|\nabla|}{\langle\nabla\rangle} \sin (t|\nabla|) u_{0}+\frac{\cos (t|\nabla|)}{\langle\nabla\rangle} u_{1},
$$

we state the following proposition, which plays an important role in obtaining the probabilistic a priori bound on the solution to the equation (3.2). This is also the key probabilistic estimate in Oh-Pocovnicu's approach to almost sure global well-posedness of energy critical wave equation in [17].

Proposition $2.7([17],[4])$. Assume $s>0$. Let $\left(u_{0}, u_{1}\right) \in \mathcal{H}^{s}\left(\mathbb{T}^{3}\right)$ be given by the series (2.4) with all coefficients real and $\left(u_{0}^{\omega}, u_{1}^{\omega}\right)$ be randomized as in (2.5). And let $T>0$ and $S^{*}(t)=S(t)$ or $\tilde{S}(t)$. Then for $2 \leq r \leq \infty$, we have

$$
\begin{aligned}
& \mathbb{P}\left(\left\|S^{*}(t)\left(u_{0}^{\omega}, u_{1}^{\omega}\right)\right\|_{L_{t}^{\infty} L_{x}^{r}\left([0, T] \times \mathbb{T}^{3}\right)}>\lambda\right) \\
& \quad \leq C \exp \left(-c \frac{\lambda^{2}}{\max \left(1, T^{2}\right)\left\|\left(u_{0}, u_{1}\right)\right\|_{\mathcal{H}^{\varepsilon}}^{2}}\right)
\end{aligned}
$$

for any $\varepsilon>0$ smaller than $s$, where the constants $C$ and $c$ depend only on $r$ and $\varepsilon$.

The proof of Proposition 2.7 runs the same as what T. Oh and O. Pocovnicu did in [17]. However, by viewing $\left\langle\partial_{t}\right\rangle^{\varepsilon}=\langle\nabla\rangle^{\varepsilon}$ when acting on $e^{ \pm i t \sqrt{-\Delta}} u_{0}$, we can prove Proposition 2.7 by the trick of losing some derivatives in space-time. See [4] for more details.

\section{Probabilistic analysis of NLW}

We first look at the truncated equation

$$
\left\{\begin{array}{l}
\left(\partial_{t}^{2}-\Delta\right) u_{N}+\left|u_{N}\right|^{p-1} u_{N}=0 \\
\left(u_{N}, \partial_{t} u_{N}\right)=\left(P_{\leq N} u_{0}, P_{\leq N} u_{1}\right)
\end{array}\right.
$$

As the initial data $\left(P_{\leq N} u_{0}, P_{\leq N} u_{1}\right)$ is smooth for any data $\left(u_{0}, u_{1}\right) \in \mathcal{H}^{s}$ with $s>0$, the equation (3.1) has a global smooth solution. In order to study the contributions of the low-frequency portion of the initial data, we rewrite (3.1) 
equivalently as

$$
\left\{\begin{array}{l}
\left(\partial_{t}^{2}-\Delta\right) v_{N}+\left|v_{N}+z_{N}\right|^{p-1}\left(v_{N}+z_{N}\right)=0 \\
\left(v_{N}, \partial_{t} v_{N}\right)=(0,0)
\end{array}\right.
$$

where $z_{N}=S(t)\left(P_{\leq N} u_{0}, P_{\leq N} u_{1}\right)$ is the free wave propagation of $\left(P_{\leq N} u_{0}, P_{\leq N} u_{1}\right)$. The following probabilistic a priori estimate of solutions to the equation (3.2) is the main result in this section.

Proposition 3.1. Let $s \in\left(\frac{p-3}{p-1}, 1\right)$ and $N \geq 1$ dyadic. Given $T, \varepsilon>0$, there exists $\tilde{\Omega}_{N, T, \varepsilon} \subset \Omega$ such that

(i) $\mathbb{P}\left(\tilde{\Omega}_{N, T, \varepsilon}^{c}\right)<\varepsilon$,

(ii) there exists a finite constant $C\left(T, \varepsilon,\left\|\left(u_{0}, u_{1}\right)\right\|_{\mathcal{H}^{s}}\right)$, independent of $N$, such that the following energy bound holds

$$
\sup _{t \in[0, T]}\left\|\left(v_{N}^{\omega}(t), \partial_{t} v_{N}^{\omega}(t)\right)\right\|_{\mathcal{H}^{1}} \leq C\left(T, \varepsilon,\left\|\left(u_{0}, u_{1}\right)\right\|_{\mathcal{H}^{s}}\right)
$$

for any solutions $v_{N}^{\omega}$ to $(3.2)$ with $\omega \in \tilde{\Omega}_{N, T, \varepsilon}$.

REMARK 3.2. Indeed, we can even choose the set $\tilde{\Omega}_{N, T, \varepsilon}$ independent of $N$, which is just a careful application of Proposition 2.6 and Proposition 2.7.

Proof. We argue in the same way as Oh-Pocovnicu did in [17]. First observe that then there exists some constant

$$
\left\|v_{N}^{\omega}\right\|_{L^{2}} \leq c\left\|v_{N}^{\omega}\right\|_{L^{p+1}} \leq c E\left(v_{N}^{\omega}\right)^{\frac{1}{p+1}}
$$

where $E(u)(t)$ is the energy function associated to the equation (1.1), and is defined by

$$
E(u)(t)=\frac{1}{2} \int_{\mathbb{T}^{3}}|\nabla u|^{2}+|u|^{2}+\left|\partial_{t} u\right|^{2}+\frac{1}{p+1} \int_{\mathbb{T}^{3}}|u|^{p+1} d x .
$$

Now if we have

$$
\sup _{t \in[0, T]} E\left(v_{N}^{\omega}\right) \leq C
$$

then we would have

$$
\sup _{t \in[0, T]}\left\|\left(v_{N}^{\omega}(t), \partial_{t} v_{N}^{\omega}(t)\right)\right\|_{\mathcal{H}^{1}}^{2} \leq\left(C+C^{\frac{2}{p+1}}\right) .
$$

Consequently, we only need to prove (3.4).

As above $z_{N}(t)=S(t)\left(P_{\leq N} u_{0}, P_{\leq N} u_{1}\right)$ and we let $\tilde{z}_{N}:=\frac{\partial_{t} z_{N}}{\langle\nabla\rangle}$. Let $\delta>0$ sufficiently small such that $\frac{p-3}{p-1}+\delta<s$. For fixed $T, \varepsilon>0$ we define $\tilde{\Omega}_{N, T, \varepsilon}$ by

$$
\begin{aligned}
\tilde{\Omega}_{N, T, \varepsilon}:= & \left\{\omega:\left\|z_{N}^{\omega}\right\|_{L_{T, x}^{2 p}}+\left\|z_{N}^{\omega}\right\|_{L_{T}^{\infty} L_{x}^{p+1}}\right. \\
& \left.+\left\|z_{N}^{\omega}\right\|_{L_{t}^{\infty} L^{\frac{4(p+1)}{5-p}}}^{2}+\left\|\langle\nabla\rangle^{s-} \tilde{z}_{N}^{\omega}\right\|_{L_{T, x}^{\infty}} \leq \lambda\right\},
\end{aligned}
$$


where $\lambda=\lambda\left(T, \varepsilon,\left\|\left(u_{0}, u_{1}\right)\right\|_{\mathcal{H}^{s}}\right)>0$ is chosen such that $\mathbb{P}\left(\tilde{\Omega}_{N, T, \varepsilon}^{c}\right)<\varepsilon$. The existence of $\tilde{\Omega}_{N, T, \varepsilon}$ is guaranteed by Lemma 2.6 and Lemma 2.7.

In the sequel, we are going to prove

$$
\sup _{t \in[0, T]} E\left(v_{N}^{\omega}(t)\right) \leq C\left(T, \varepsilon,\left\|\left(u_{0}, u_{1}\right)\right\|_{\mathcal{H}^{s}}\right)
$$

for $\omega \in \tilde{\Omega}_{N, T, \varepsilon}$. In the following of this section, we suppress the index $N$ for the solution $v_{N}$ to the equation (3.2). We will also do this for the linear evolution $z_{N}(t)$. In order to achieve the energy bound (3.5), we differentiate the expression of the energy and calculate

$$
\begin{aligned}
\frac{d}{d t} E(v)(t) & =\int_{\mathbb{T}^{3}} \partial_{t} v\left(\partial_{t}^{2} v-\Delta v+|v|^{p-1} v\right) d x \\
& =-\int_{\mathbb{T}^{3}} \partial_{t} v\left(|v+z|^{p-1}(v+z)-|v|^{p-1} v\right) d x \\
& =-\int_{\mathbb{T}^{3}} \partial_{t} v\left(p|v|^{p-1} z+p(p-1)|v+\theta z|^{p-2} z^{2}\right) d x
\end{aligned}
$$

where in the last equality we have used differential mean value equality with $\theta \in[0,1]$. By integrating in time, we have

$$
\begin{aligned}
E(v)(t)= & E(v)(0)-\int_{0}^{t} \int_{\mathbb{T}^{3}} \partial_{t} v\left(t^{\prime}\right)\left[p z\left(t^{\prime}\right)\left|v\left(t^{\prime}\right)\right|^{p-1}\right. \\
& \left.+p(p-1)\left|v\left(t^{\prime}\right)+\theta z\left(t^{\prime}\right)\right|^{p-2} z\left(t^{\prime}\right)^{2}\right] d t^{\prime} d x \\
= & -\int_{\mathbb{T}^{3}} \int_{0}^{t} z\left(t^{\prime}\right) \partial_{t}\left[|v|^{p-1} v\left(t^{\prime}\right)\right] d t^{\prime} d x \\
& -\int_{0}^{t} \int_{\mathbb{T}^{3}} \partial_{t} v\left(t^{\prime}\right) p(p-1)\left|v\left(t^{\prime}\right)+\theta z\left(t^{\prime}\right)\right|^{p-2} z\left(t^{\prime}\right)^{2} d t^{\prime} d x \\
= & : I(t)+I I(t) .
\end{aligned}
$$

Noticing that

$$
|| v+\left.\theta z\right|^{p-2} z^{2} \mid \leq c\left(|v|^{p-2} z^{2}+|z|^{p}\right)
$$

where $c$ is a constant depending only on $p$, we have

$$
\begin{aligned}
|I I(t)| \leq & \int_{0}^{t}\left\|\partial_{t} v\left(t^{\prime}\right)\right\|_{L^{2}}\left\|v\left(t^{\prime}\right)\right\|_{L^{p+1}}^{p-2}\|z\|_{L^{\frac{4(p+1)}{5-p}}}^{2}\left(t^{\prime}\right) \\
& +\int_{0}^{t}\left\|\partial_{t} v\left(t^{\prime}\right)\right\|_{L^{2}}\left\|z\left(t^{\prime}\right)\right\|_{L^{2 p}}^{2 p} d t^{\prime} \\
\leq & \left(1+\|z\|_{L_{t}^{\infty} L^{\frac{4(p+1)}{5-p}}}^{2}\right) \int_{0}^{t} \max \left(E(v)\left(t^{\prime}\right), E(v)^{\frac{3(p-1)}{2(p+1)}}\right) d t^{\prime}+\|z\|_{L_{T}^{4 p} L_{x}^{2 p}}^{2 p}
\end{aligned}
$$


Thanks to the assumption $p<5$, we have that $\frac{3 p-3}{2 p+2} \leq 1$. And hence we only need to consider

$$
|I I(t)| \leq\left(1+\|z\|_{L_{t}^{\infty} L^{\frac{4(p+1)}{5-p}}}^{2}\right) \int_{0}^{t} E(v)\left(t^{\prime}\right) d t^{\prime}+\|z\|_{L_{T}^{4 p} L_{x}^{2 p}}^{2 p} .
$$

First, we are going to deal with the term $I(t)$. As $v(0)=0$ and $v=v_{N}^{\omega}$ is smooth, both in $t$ and $x$, integrating by parts, we have

$$
I(t)=-\int_{\mathbb{T}^{3}} z(t)|v|^{p}+\int_{\mathbb{T}^{3}} \int_{0}^{t} \partial_{t} z\left(t^{\prime}\right)\left|v\left(t^{\prime}\right)\right|^{p} d t^{\prime} d x=: I_{1}(t)+I_{2}(t) .
$$

As for the first term $I_{1}(t)$, we have

$$
\left|I_{1}(t)\right| \leq a\|v(t)\|_{L^{p+1}}^{p+1}+a^{-p}\|z(t)\|_{L^{p+1}}^{p+1} \leq a E(v)(t)+a^{-p}\|z\|_{L_{T}^{\infty} L_{x}^{p+1}}^{p+1},
$$

where $a$ is a small constant, to be chosen later.

Then we turn to bound the term $I_{2}(t)$. To do this, we need the following lemma.

Lemma 3.3. Let $v, \tilde{z}$ as above. Then there exists some constant $C$ such that,

$$
\left.\left|\int_{\mathbb{T}^{3}}\right| v\right|^{p-1} v\langle\nabla\rangle \tilde{z} d x \mid \leq C\left[\left(\left\|\langle\nabla\rangle^{s-} \tilde{z}\right\|_{L_{x}^{\infty}}+1\right) E(v)(t)+\left\|\langle\nabla\rangle^{s-} \tilde{z}\right\|_{L_{x}^{\infty}}^{p+1}\right],
$$

where $s-:=s-\delta$ for any sufficiently small, positive $\delta$.

Proof. Denote $P_{j}$ the Littlewood-Paley projection onto the dyadic $2^{j}$ for $j \in \mathbb{N}^{+}$. Then we have

$$
\int_{\mathbb{T}^{3}}|v|^{p-1} v\langle\nabla\rangle \tilde{z} d x \sim \sum_{k=-1}^{k=1} \sum_{j \geq 0} \int_{\mathbb{T}^{3}} P_{j+k}\left(|v|^{p-1} v\right) P_{j}(\langle\nabla\rangle z) d x .
$$

Notice that the contribution of the summation over $k=-1,0,1$ can be bounded by that of the case $k=0$, so in the following we will omit the summatin over the index $k$ and sometimes omit the index $k$ directly.

For the low frequency case $j \leq 2$, we have

$$
\left|\int_{\mathbb{T}^{3}} P_{j+k}\left(|v|^{p-1} v\right) P_{j}(\langle\nabla\rangle \tilde{z}) d x\right| \leq\left\|\langle\nabla\rangle^{s-} \tilde{z}\right\|_{L_{x}^{\infty}}\|v\|_{L^{p+1}}^{p} .
$$

A further application of Hölder inequality, we have

$$
\left|\sum_{j \leq 2} \int_{\mathbb{T}^{3}} P_{j+k}\left(|v|^{p-1} v\right) P_{j}(\langle\nabla\rangle \tilde{z})\right| \leq\left\|\langle\nabla\rangle^{s-} \tilde{z}\right\|_{L_{x}^{\infty}}^{p+1}+E(v)(t) .
$$

For the high frequency portion $j>2$, we split the nonlinear part $P_{j}\left(|v|^{p-1} v\right)$ into the small value part and large value part. Precisely, we introduce a bump 
function $\chi: \mathbb{R}^{+} \rightarrow[0,1]$, which takes its value 1 on $[0,1]$ and vanishes outside $[0,2]$, then we split

$$
\begin{aligned}
P_{j}\left(|v|^{p-1} v\right) & =P_{j}\left(|v|^{p-1} v \chi\left(\frac{v^{2}}{\lambda_{j}^{2}}\right)\right)+P_{j}\left(|v|^{p-1} v\left(1-\chi\left(\frac{v^{2}}{\lambda_{j}^{2}}\right)\right)\right) \\
& =: I_{21}+I_{22},
\end{aligned}
$$

where $\lambda_{j}$ is a sequence of numbers to be chosen later.

For small values of $v$, by Hölder inequality, Bernstein's inequality and the boundedness of Riesz transformation, we can do the following calculations

$$
\begin{aligned}
\left|\int_{\mathbb{T}^{3}} P_{j}\left(|v|^{p-1} v \chi\right) P_{j}(\langle\nabla\rangle z) d x\right| & \left|\int_{\mathbb{T}^{3}} P_{j}\left(|v|^{p-1} v \chi\right) \nabla \cdot \nabla^{-1} P_{j}(\langle\nabla\rangle z) d x\right| \\
= & \left|\int_{\mathbb{T}^{3}} \nabla P_{j}\left(|v|^{p-1} v \chi\right) \nabla^{-1} P_{j}(\langle\nabla\rangle z) d x\right| \\
& \lesssim 2^{-j(s-)}\left\|\langle\nabla\rangle^{s-\tilde{z}}\right\|_{L_{x}^{\infty}}\left\|P_{j}\left(\nabla\left(|v|^{p-1} v \chi\right)\right)\right\|_{L^{1}} \\
& \lesssim 2^{-j(s-)}\left\|\langle\nabla\rangle^{s-} \tilde{z}\right\|_{L_{x}^{\infty}}\left\|v^{p-1} \nabla v \chi\right\|_{L_{x}^{1}} \\
& \lesssim 2^{-j(s-)}\left\|\langle\nabla\rangle^{s-} \tilde{z}\right\|_{L_{x}^{\infty}}\left\||v|^{p-1-\frac{p+1}{2}} \chi\right\|_{L_{x}^{\infty}} \\
& \times\|\nabla v\|\left\|_{L^{2}}\right\||v|^{\frac{p+1}{2}} \|_{L^{2}} \\
& \lesssim 2^{-j(s-)}\left\|\langle\nabla\rangle^{s-} \tilde{z}\right\|_{L_{x}^{\infty}} \lambda_{j}^{\frac{p-3}{2}} E(v)(t) .
\end{aligned}
$$

To guarantee the convergence of the series $\sum_{j \geq 2} 2^{-j(s-)} \lambda_{j}^{\frac{p-3}{2}}$, we choose $\lambda_{j}=2^{a j}$ with $a \in\left(0, \frac{2 s-}{p-3}\right)$. And in this case, we have

$$
\left|\sum_{j>2} \int_{\mathbb{T}^{3}} P_{j}\left(|v|^{p-1} v \chi\right) P_{j}(\langle\nabla\rangle z) d x\right| \lesssim\left\|\langle\nabla\rangle^{s-} \tilde{z}\right\|_{L_{x}^{\infty}} E(v)(t)
$$

provided that the Sobolev regularity index $s$ is positive.

For the case $v$ is large, we first consider the case $[p]$ is odd. By denoting $\alpha=p-[p]$, we do the following calculations

$$
\begin{aligned}
& \left|\int_{\mathbb{T}^{3}} P_{j}\left(|v|^{p-1} v(1-\chi)\right) P_{j}(\langle\nabla\rangle z) d x\right| \\
& \quad \leq\left|\int_{\mathbb{T}^{3}} P_{j}\left(\sum_{j_{1}, j_{2}, \ldots, j_{[p]-1}, \gamma}\left(\prod_{i=1}^{[p]-1} P_{j_{i}} v\right) P_{\gamma}\left(v|v|^{\alpha}(1-\chi)\right)\right) P_{j}(\langle\nabla\rangle \tilde{z}) d x\right| \\
& \quad \leq\left\|P_{j}\langle\nabla\rangle^{s-\tilde{z}}\right\|_{L_{x}^{\infty}} 2^{j(1-(s-))} \sum_{j_{1}, \ldots, j_{[p]-1}, \gamma}\left\|\left(\prod_{i=1}^{[p]-1} P_{j_{i}} v\right) P_{\gamma}\left(v|v|^{\alpha}(1-\chi)\right)\right\|_{L_{x}^{1}} \\
& \quad \leq M_{j}+N_{j},
\end{aligned}
$$


where

$$
\begin{aligned}
M_{j}:= & \left\|P_{j}\langle\nabla\rangle^{s-} \tilde{z}\right\|_{L_{x}^{\infty}} 2^{j(1-(s-))} \\
& \times \sum_{j_{1} \geq \max \left(j_{2}, \ldots, j_{[p]-1}, \gamma\right)}\left\|\left(\prod_{i=1}^{[p]-1} P_{j_{i}} v\right) P_{\gamma}\left(v|v|^{\alpha}(1-\chi)\right)\right\|_{L_{x}^{1}}
\end{aligned}
$$

and

$$
\begin{aligned}
N_{j}:= & \left\|P_{j}\langle\nabla\rangle^{s-} \tilde{z}\right\|_{L_{x}^{\infty}} 2^{j(1-(s-))} \\
& \times \sum_{\gamma \geq \max \left(j_{1}, \ldots, j_{[p]-1}\right)}\left\|\left(\prod_{i=1}^{[p]-1} P_{j_{i}} v\right) P_{\gamma}\left(v|v|^{\alpha}(1-\chi)\right)\right\|_{L_{x}^{1}} .
\end{aligned}
$$

(1) To control $M_{j}$ : observe that if $j \gg j_{1}$, we should have that $M_{j}=0$. And hence, we have

$$
\begin{aligned}
& \sum_{j>2} M_{j} \leq \sum_{j>2}\left\|P_{j}\langle\nabla\rangle^{s-} \tilde{z}\right\|_{L_{x}^{\infty}} 2^{j(1-(s-))} \\
& \times \sum_{\substack{j_{1} \geq j_{2}, \ldots, j_{[p]-1} \\
j_{1} \geq \gamma \\
j_{1}+[p] \geq j}}\left\|\left(\prod_{i=1}^{[p]-1} P_{j_{i}} v\right) P_{\gamma}\left(v|v|^{\alpha}(1-\chi)\right)\right\|_{L_{x}^{1}} \\
& \leq \sum_{j>2}\left\|P_{j}\langle\nabla\rangle^{s-} \tilde{z}\right\|_{L_{x}^{\infty}} 2^{j(1-(s-))} \\
& \times \sum_{\substack{j_{1} \geq j_{2}, \ldots, j_{[p]-1} \\
j_{1} \geq \gamma \\
j_{1}+[p] \geq j}}\left\|P_{j_{1}} v\right\|_{L_{x}^{\frac{p+1}{2}}}\left\|\prod_{i=2}^{[p]-1} P_{j_{i}} v\right\|\left\|_{\substack{\frac{p+1}{L_{x}}[p-2 \\
[p]}}\right\| P_{\gamma}\left(v|v|^{\alpha}(1-\chi)\right) \|_{L_{x}^{\frac{p+1}{1+\alpha}}} \\
& \leq \sum_{j>2}\left\|P_{j}\langle\nabla\rangle^{s-} \tilde{z}\right\|_{L_{x}^{\infty}} 2^{j(1-(s-))} \sum_{j_{1}+[p] \geq j}\left\|P_{j_{1}} v\right\|_{L_{x}^{\frac{p+1}{2}}}\|v\|_{L_{x}^{p+1}}^{p-1} \\
& \leq \sum_{j>2}\left\|P_{j}\langle\nabla\rangle^{s-} \tilde{z}\right\|_{L_{x}^{\infty}} 2^{j(1-(s-))} \sum_{j_{1}+[p] \geq j}\left\|P_{j_{1}} v\right\|_{L^{2}}^{\frac{2}{p-1}}\left\|P_{j_{1}} v\right\|_{L^{p+1}}^{\frac{p-3}{p-1}}\|v\|_{L_{x}^{p+1}}^{p-1} \\
& \lesssim \sum_{j>2}\left\|P_{j}\langle\nabla\rangle^{s-} \tilde{z}\right\|_{L_{x}^{\infty}} 2^{j(1-(s-))} \sum_{j_{1}+[p] \geq j} 2^{-j_{1} \frac{2}{p-1}}\left\|P_{j_{1}} \nabla v\right\|_{L^{2}}^{\frac{2}{p-2}}\|v\|_{L_{x}^{p+1}}^{p-\frac{2}{p-1}} \\
& \lesssim \sum_{j>2}\left\|\langle\nabla\rangle^{s-} \tilde{z}\right\|_{L_{x}^{\infty}} 2^{j(1-(s-))} 2^{-j\left(\frac{2}{p-1}-\right)} E(v),
\end{aligned}
$$


where in the second last inequality, we have used the boundedness of Riesz transformation. Consequently, the last series converges provided

$$
s>\frac{p-3}{p-1} .
$$

And in this case, we have

$$
\sum_{j>2} M_{j} \lesssim\left\|\langle\nabla\rangle^{s-} \tilde{z}\right\|_{L_{x}^{\infty}} E(v)(t) .
$$

(2) To control $N_{j}$ : the same observation as in controlling $M_{j}$ allows us to only need to deal with the case $\gamma+[p] \geq j$. Then

$$
\begin{aligned}
& \sum_{j>2} N_{j}=\sum_{j>2}\left\|P_{j}\langle\nabla\rangle^{s-} \tilde{z}\right\|_{L_{x}^{\infty}} 2^{j(1-(s-))} \\
& \times \sum_{\substack{\gamma \geq j_{1}, \ldots, j_{[p]-1} \\
\gamma+[p] \geq j}}\left\|\left(\prod_{i=1}^{[p]-1} P_{j_{i}} v\right) P_{\gamma}\left(v|v|^{\alpha}(1-\chi)\right)\right\|_{L_{x}^{1}} \\
& =\sum_{j>2}\left\|P_{j}\langle\nabla\rangle^{s-} \tilde{z}\right\|_{L_{x}^{\infty}} 2^{j(1-(s-))} \\
& \times \sum_{\substack{\gamma \geq j_{1}, \ldots, j_{[p]-1} \\
\gamma+[p] \geq j}}\left\|P_{\gamma}\left(v|v|^{\alpha}(1-\chi)\right)\right\|_{L^{\frac{p+1}{2+\alpha}}}\left\|\prod_{i=1}^{[p]-1} P_{j_{i}} v\right\|_{L_{x}^{\frac{p+1}{[p]-1}}} \\
& \leq \sum_{j>2}\left\|\langle\nabla\rangle^{s-} \tilde{z}\right\|_{L_{x}^{\infty}} 2^{j(1-(s-))} \\
& \times \sum_{\gamma+[p] \geq j}\left\|P_{\gamma}\left(v|v|^{\alpha}(1-\chi)\right)\right\|\left\|_{L_{x}^{\frac{2(p+1)}{p+1+2 \alpha}}}^{\frac{2}{p-1}}\right\| P_{\gamma}\left(v|v|^{\alpha}(1-\chi)\right)\left\|_{L_{x}^{\frac{p+1}{1+\alpha}}}^{\frac{p-3}{p-1}}\right\| v \|_{L_{x}^{p+1}}^{[p]-1} \\
& \leq \sum_{j>2}\left\|\langle\nabla\rangle^{s-} \tilde{z}\right\|_{L_{x}^{\infty}} 2^{j(1-(s-))} \\
& \times \sum_{\gamma+[p] \geq j}\left\|P_{\gamma}\left(v|v|^{\alpha}(1-\chi)\right)\right\|_{L_{x}^{\frac{2(p+1)}{p+1+2 \alpha}}}^{\frac{2}{p-1}}\|v\|_{L_{x}^{p+1}}^{p-\frac{2(1+\alpha)}{p-1}} \\
& \lesssim \sum_{j>2}\left\|\langle\nabla\rangle^{s-} \tilde{z}\right\|_{L_{x}^{\infty}} 2^{j(1-(s-))} \\
& \times \sum_{\gamma+[p] \geq j} 2^{-\gamma \frac{2}{p-1}}\left\|\nabla P_{\gamma}\left(v|v|^{\alpha}(1-\chi)\right)\right\|_{L_{x}^{\frac{2(p+1)}{p+1+2 \alpha}}}^{\frac{2}{p-1}}\|v\|_{L_{x}^{p+1}}^{p-\frac{2(1+\alpha)}{p-1}}
\end{aligned}
$$

where in the last inequality, we have used the boundedness of Riesz transformation. Since

$$
\nabla P_{\gamma}\left(v|v|^{\alpha}(1-\chi)\right) \sim \nabla v|v|^{\alpha}
$$


we have that

$$
\begin{aligned}
\sum_{j>2} N_{j} \lesssim & \sum_{j>2}\left\|\langle\nabla\rangle^{s-\tilde{z}}\right\|_{L_{x}^{\infty}} 2^{j(1-(s-))} \\
& \times \sum_{\gamma+[p] \geq j} 2^{-\gamma \frac{2}{p-1}}\left\|\nabla v \times|v|^{\alpha}\right\|_{L_{x}^{\frac{2(p+1)}{p+1+2 \alpha}}\|v\|_{L_{x}^{p+1}}^{p-\frac{2(1+\alpha)}{p-1}}}^{\frac{2}{p+1}} \\
\lesssim & \sum_{j>2}\left\|\langle\nabla\rangle^{s-\tilde{z}}\right\|_{L_{x}^{\infty}} 2^{j(1-(s-))} \\
& \times \sum_{\gamma+[p] \geq j} 2^{-\gamma \frac{2}{p-1}}\|\nabla v\|_{L^{2}}^{\frac{2}{p-1}}\left\||v|^{\alpha}\right\|_{L_{x}^{\frac{p+1}{\alpha}}}^{\frac{2}{p-1}}\|v\|_{L_{x}^{p+1}}^{p-\frac{2(1+\alpha)}{p-1}} \\
\lesssim & \sum_{j>2}\left\|\langle\nabla\rangle^{s-\tilde{z}}\right\|_{L_{x}^{\infty}} 2^{j(1-(s-))} \sum_{\gamma+[p] \geq j} 2^{-\gamma \frac{2}{p-1}} E(v) \\
\lesssim & \sum_{j>2}\left\|\langle\nabla\rangle^{s-\tilde{z}}\right\|_{L_{x}^{\infty}} 2^{j(1-(s-))} 2^{\left(-j \frac{2}{p-1}\right)+} E(v) .
\end{aligned}
$$

Thus the last series converges provided that

$$
s>\frac{p-3}{p-1} .
$$

And in this case we have

$$
\sum_{j>2} N_{j} \lesssim\left\|\langle\nabla\rangle^{s-} \tilde{z}\right\|_{L_{x}^{\infty}} E(v)(t)
$$

For the case $[p]$ is even, we should replace the expression $P_{j}\left(|v|^{p-1} v\right)=$ $\sum P_{j}\left(\prod_{i=1}^{[p]-1} P_{j_{i}} v P_{\gamma}\left(v|v|^{\alpha}\right)\right)$ in the case that $[p]$ is odd by the expression $P_{j}\left(|v|^{p-1} v\right)=\sum P_{j}\left(\prod_{i=1}^{[p]-2} P_{j_{i}} v P_{\gamma}\left(v|v|^{1+\alpha}\right)\right)$, and do the same calculations as above with some different Hölder indices.

Now, in our situation, it is only left to prove the case $\alpha=0$, which is just the case $p=4$. Indeed, this case is much easier to check.

By collecting the bounds (3.9), (3.10), (3.11) and (3.12), we can close the proof of Lemma 3.3.

As a consequence of Lemma 3.3, by the fact that $\partial_{t} z(t)=\langle\nabla\rangle \tilde{z}$, we have

$$
\left|I_{2}\right| \leq \int_{0}^{t}\left(\left\|\langle\nabla\rangle^{s-} \tilde{z}\right\|_{L_{x}^{\infty}}\left(t^{\prime}\right)\right)^{p+1}\left(1+\left\|\langle\nabla\rangle^{s-} \tilde{z}\right\|_{L_{x}^{\infty}}\left(t^{\prime}\right)\right) E(v)\left(t^{\prime}\right) d t^{\prime} .
$$

Finally, by collecting the estimates (3.6), (3.8) and (3.13) together, with a sufficiently small, and using Gronwall's lemma, one can finish the proof of Proposition 3.1. 


\section{Deterministic analysis of NLW}

Using energy and Strichartz estimates, we can establish the following lemma, which is the key deterministic step in constructing solutions for the problem (1.1).

LEMMA 4.1. Let $p \in(3,5)$ and suppose that $\left(v_{0}, v_{1}\right) \in \mathcal{H}^{1}$ is bounded by some constant $C,{ }^{2}$ then for the wave equation

$$
\left\{\begin{array}{l}
\left(\partial_{t}^{2}-\Delta\right) v+|v+f|^{p-1}(v+f)=0 \\
\left.\left(v, \partial_{t} v\right)\right|_{t=t_{0}}=\left(v_{0}, v_{1}\right) \in \mathcal{H}^{1}\left(\mathbb{T}^{3}\right)
\end{array}\right.
$$

there exists $t_{*}>0$, such that the equation (4.1) has a unique solution in $\left(C\left(\left[t_{0}, t_{0}+t_{*}\right] ; H_{x}^{1}\right) \cap L_{t}^{\frac{2 p}{p-3}}\left(I ; L_{x}^{2 p}\right)\right) \times C\left(\left[t_{0}, t_{0}+t_{*}\right] ; L_{x}^{2}\right)=: X$, under the condition that

$$
\|f\|_{L_{t}^{\frac{2 p}{p-3}}\left(\left[t_{0}, t_{0}+t_{*}\right] ; L_{x}^{2 p}\right)} \leq K t_{*}^{\beta},
$$

where $\beta$ is some positive number.

REMARK 4.2. Thanks to the fact that $p$ is strictly less than 5 , we do not need to prove Lemma 4.1 via the stability theory for the critical NLW as Pocovnicu did in [18].

The proof of Lemma 4.1 is a standard argument via fixed point argument. As we will play this argument in the forthcoming Lemma 4.4, we omit the proof here.

Now we are going to construct solutions for the equation (1.1). By denoting $v:=u-f$ with $f=S(t)\left(u_{0}, u_{1}\right)$, then $v$ satisfies the following zero-initial data problem

$$
\left\{\begin{array}{l}
\left(\partial_{t}^{2}-\Delta\right) v+|v+f|^{p-1}(v+f)=0, \\
\left.\left(v, \partial_{t} v\right)\right|_{t=0}=(0,0) .
\end{array}\right.
$$

The following deterministic result, allows us to draw an a priori energy bound of solution $v$ to the equation (4.3) with $f=S(t)\left(u_{0}^{\omega}, u_{1}^{\omega}\right)$ from that of solution $v_{N}$ to the truncated equation (3.2).

Proposition 4.3. Let $f_{N}:=P_{\leq N} f$ denote the projection onto the first $N$-Fourier modes of the given function $f$ and $v_{N}$ be the solution to the truncated wave equation (3.2). Given finite $T>0$, assume the following conditions hold:

(i) There exists $K>0$ for some $\beta>0$ such that

$$
\|f\|_{L_{t}^{\frac{2 p}{p-3}} L_{x}^{2 p}\left(I \times \mathbb{T}^{3}\right)} \leq K|I|^{\beta}
$$

for any compact interval $I \subset[0, T]$.

\footnotetext{
${ }^{2}$ In the forthcoming application, the data is chosen to be bounded by the constant $C$ as in (3.3).
} 
(ii) For each dyadic $N \geq 1$, the solution $v_{N}$ to $(3.2)$ exists on $[0, T]$ and satisfies uniformly the following a priori energy bound

$$
\sup _{N} \sup _{t \in[0, T]} \|\left(v_{N}(t), \partial_{t} v_{N}(t) \|_{\mathcal{H}^{1}\left(\mathbb{T}^{3}\right)}<C_{0}(T)<\infty .\right.
$$

(iii) There holds for any dyadic $N \geq 1$ and some $\alpha>0$

$$
\left\|f-f_{N}\right\|_{L_{[0, T]}^{\frac{2 p}{p-3}} L_{x}^{2 p}} \leq C_{1}(T) N^{-\alpha} .
$$

Then there exists a unique solution $\left(v, \partial_{t} v\right) \in C\left([0, T] ; \mathcal{H}^{1}\left(\mathbb{T}^{3}\right)\right)$ to (4.3) satisfying

$$
\sup _{t \in[0, T]}\left\|\left(v(t), \partial_{t} v(t)\right)\right\|_{\mathcal{H}^{1}\left(\mathbb{T}^{3}\right)}<2 C_{0}(T)<\infty .
$$

Proof. To prove Proposition 4.3, we need the following lemma, which states that we can solve simultaneously, on some time interval $\left[t_{0}, t_{0}+t_{*}\right]$ for any $t_{0} \in[0, T)$, the following two equations

$$
\left\{\begin{array}{l}
\left(\partial_{t}^{2}-\Delta\right) v_{N}+\left|v_{N}+f_{N}\right|^{p-1}\left(v_{N}+f_{N}\right)=0 \\
\left.\left(v_{N}, \partial_{t} v_{N}\right)\right|_{t=t_{0}}=\left(v_{N}\left(t_{0}\right), \partial_{t} v_{N}\left(t_{0}\right)\right)
\end{array}\right.
$$

and

$$
\left\{\begin{array}{l}
\left(\partial_{t}^{2}-\Delta\right) v+|f+v|^{p-1}(f+v)=0 \\
\left.\left(v, \partial_{t} v\right)\right|_{t=t_{0}}=\left(v\left(t_{0}\right), \partial_{t} v\left(t_{0}\right)\right)
\end{array}\right.
$$

Lemma 4.4. Assume there hold (4.4), (4.5), (4.6). Assume also there holds for any $t_{0} \in[0, T)$

$$
\sup _{t \in\left[0, t_{0}\right]}\left\|\left(v, \partial_{t} v\right)\right\|_{\mathcal{H}^{1}}<2 C_{0}(T)<\infty
$$

where $C_{0}(T)$ is the same constant as in the assumption (4.5). Then there exist a sufficiently large $N_{1}$ and a positive time $t_{*}=t_{*}\left(C_{0}, K, N_{1}\right)>0$ such that, for all $N \geq N_{1}$, on the time interval $I=\left[t_{0}, t_{0}+t_{*}\right]$, we can solve simultaneously the equations (4.8) and (4.9). Moreover, if we denote these solutions by $v_{n}$ and $v$ respectively, we have

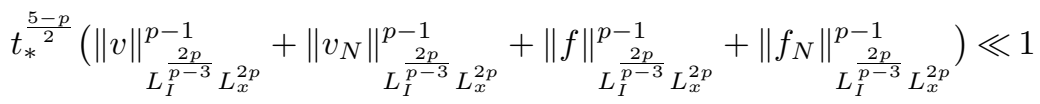

for all $N \geq N_{1}$.

Proof. We will use fixed point argument to prove this lemma. As this is standard, we only outline the main steps here and give precisely these estimates we are going to use in the following of this section. Let $X$ be the space as in Lemma 4.1. Define the maps $L_{1}$ on $B\left(0, R_{1}\right) \subset X$ and $L_{2}$ on 
$B\left(0, R_{2}\right)$ respectively, as:

$$
\begin{aligned}
& L_{1}: u_{N} \in B\left(0, R_{1}\right) \longmapsto v_{N}, \\
& L_{2}: u \in B\left(0, R_{2}\right) \longmapsto v,
\end{aligned}
$$

where $v_{N}$ and $v$ solves respectively, the equations

$$
\left\{\begin{array}{l}
\left(\partial_{t}^{2}-\Delta\right) v_{N}+\left|u_{N}+f_{N}\right|^{p-1}\left(u_{N}+f_{N}\right)=0 \\
\left.\left(v_{N}, \partial_{t} v_{N}\right)\right|_{t=t_{0}}=\left(v_{N}\left(t_{0}\right), \partial_{t} v_{N}\left(t_{0}\right)\right)
\end{array}\right.
$$

and

$$
\left\{\begin{array}{l}
\left(\partial_{t}^{2}-\Delta\right) v+|f+u|^{p-1}(f+u)=0 \\
\left.\left(v, \partial_{t} v\right)\right|_{t=t_{0}}=\left(v\left(t_{0}\right), \partial_{t} v\left(t_{0}\right)\right)
\end{array}\right.
$$

By (4.4) and (4.6), we have

$$
\left\|f_{N}\right\|_{L_{I}^{\frac{2 p}{p-3}} L_{x}^{2 p}} \leq K|I|^{\beta}+C_{1}(T) N^{-\alpha} .
$$

In order to show $L_{1}$ to be a contracting map onto $B\left(0, R_{1}\right)$, by using energy and Strichartz estimates, we have for $\left(v_{N}, \partial_{t} v_{N}\right) \in B\left(0, R_{1}\right)$ and $u, \tilde{u} \in$ $B\left(0, R_{1}\right)$ the following estimates

$$
\begin{aligned}
\left\|L_{1} v_{N}\right\|_{X} & \lesssim\left\|\left(v_{N}\left(t_{0}\right), \partial_{t} v_{N}\left(t_{0}\right)\right)\right\|_{\mathcal{H}^{1}}+t_{*}^{\frac{5-p}{2}}\left(\left\|v_{N}\right\|_{L_{t}^{\frac{2 p}{p-3}} L_{x}^{2 p}}^{p}+\left\|f_{N}\right\|_{L_{t}^{\frac{2 p}{p-3}} L_{x}^{2 p}}^{p}\right) \\
& \lesssim\left\|\left(v_{N}\left(t_{0}\right), \partial_{t} v_{N}\left(t_{0}\right)\right)\right\|_{\mathcal{H}^{1}}+t_{*}^{\frac{5-p}{2}}\left(\left\|v_{N}\right\|_{L_{t}^{\frac{2 p}{p-3}} L_{x}^{2 p}}^{p}+K^{p} t_{*}^{p \beta}+N^{-p \alpha}\right),
\end{aligned}
$$

and

$$
\begin{aligned}
\left\|L_{1} u-L_{1} \tilde{u}\right\|_{X} \lesssim & t_{*}^{\frac{5-p}{2}}\|u-\tilde{u}\|_{X}\left(\|u\|_{X}^{p-1}+\|\tilde{u}\|_{X}^{p-1}+\|f\|_{L_{t}^{\frac{2 p}{p-3} L_{x}^{2 p}}}^{p-1}\right) \\
& \lesssim t_{*}^{\frac{5-p}{2}}\|u-\tilde{u}\|_{X} \\
& \quad \times\left(\|u\|_{X}^{p-1}+\|\tilde{u}\|_{X}^{p-1}+K^{p-1} t_{*}^{(p-1) \beta}+N^{-(p-1) \alpha}\right) .
\end{aligned}
$$

Hence we conclude that, under the assumptions that

$$
\left\{\begin{array}{l}
R_{1}=2 C_{0}(T), \\
t_{*}^{\frac{5-p}{2}} R_{1}^{p-1} \ll 1, \\
t_{*}^{\frac{5-p}{2}}\left(K^{p} t_{*}^{p \beta}+N^{-p \alpha}\right) \ll R_{1}, \\
t_{*}^{\frac{5-p}{2}}\left(K^{p-1} t_{*}^{(p-1) \beta}+N^{-(p-1) \alpha}\right) \ll 1,
\end{array}\right.
$$


the solution map $L_{1}$ is a contraction map onto $B\left(0, R_{1}\right)$. Doing similar calculations as above, we can see that under the conditions that

$$
\left\{\begin{array}{l}
R_{1}=2 C_{0}(T), \\
t_{*}^{\frac{5-p}{2}} R_{2}^{p-1} \ll 1, \\
t_{*}^{\frac{5-p}{2}} K^{p} t_{*}^{p} \ll R_{2}, \\
t_{*}^{\frac{5-p}{2}} K^{p-1} t_{*}^{p-1} \ll 1,
\end{array}\right.
$$

the solution map $L_{2}$ is also a contraction map onto $B\left(0, R_{2}\right)$.

Thus there exists sufficiently large $N_{1}=N_{1}\left(K, C_{0}(T)\right)$ such that, for all $N \geq N_{1}$, by choosing $t_{*}=c\left(K+C_{0}(T)\right)^{-\gamma}$ with $c$ and $\gamma$ small positive constants, these two assumptions hold true at the same time. And hence we can solve these two equations on the same time interval $\left[t_{0}, t_{0}+t_{*}\right]$. By choosing $t_{*}$ even smaller if necessary, we can validate the estimate (4.11).

As a consequence of the proof of Lemma 4.4, we have for the difference $w_{N}=v-v_{N}$ on the time interval $I=\left[t_{0}, t_{0}+t_{*}\right]$ :

$$
\begin{aligned}
& \left\|w_{N}\right\|_{L_{I}^{\infty} \mathcal{H}^{1}}+\left\|w_{N}\right\|_{L_{I}^{\frac{2 p}{p-3}} L_{x}^{2 p}} \\
& \quad \leq C_{2}\left\|w_{N}\left(t_{0}\right)\right\|_{\mathcal{H}^{1}}+\frac{1}{2}\left\|w_{N}\right\|_{L_{I}^{\frac{2 p}{p-3}} L_{x}^{2 p}}+\frac{1}{2}\left\|f-f_{N}\right\|_{L_{I}^{\frac{2 p}{p-3}} L_{x}^{2 p}} .
\end{aligned}
$$

Thus we have

$$
\left\|w_{N}\right\|_{L_{I}^{\infty} \mathcal{H}^{1}}+\left\|w_{N}\right\|_{L_{I}^{\frac{2 p}{p-3}} L_{x}^{2 p}} \leq C_{3}(T)\left(\left\|w_{N}\left(t_{0}\right)\right\|_{\mathcal{H}^{1}}+N^{-\alpha}\right)
$$

for all $N \geq N_{1}$.

With these estimates, we start to solve the problem (4.3) with $t_{0}=0$. As $\left\|\left(v, \partial_{t} v\right)\right\|_{\mathcal{H}^{1}}(0)=0<2 C_{0}(T)$, we can solve simultaneously the equations (4.8) and (4.9) on the time interval $I_{0}=\left[0, t_{*}\right]$, where $t_{*}$ is obtained in Lemma 4.4 and it depends only on $C_{0}(T)$ and $K$. Furthermore, by (4.14) and (4.15), we have for all $N \geq N_{1}$

$$
t_{*}^{\frac{5-p}{2}}\left(\|v\|_{L_{I_{0}}^{\frac{2 p}{p-3}} L_{x}^{2 p}}^{p-1}+\left\|v_{N}\right\|_{L_{I_{0}}^{\frac{2 p}{p-3}} L_{x}^{2 p}}^{p-1}+\|f\|_{L_{I_{0}}^{p-3} L_{x}^{2 p}}^{p-1}+\left\|f_{N}\right\|_{L_{I_{0}}^{p-3} L_{x}^{2 p}}^{p-1}\right) \ll 1,
$$

and hence

$$
\begin{aligned}
& \left\|w_{N}\right\|_{L_{I_{0}}^{\infty} \mathcal{H}^{1}}+\left\|w_{N}\right\|_{L_{I_{0}}^{\frac{2 p}{p-3}} L_{x}^{2 p}} \\
& \quad \leq C_{2}\left\|w_{N}(0)\right\|_{\mathcal{H}^{1}}+\frac{1}{2}\left\|w_{N}\right\|_{L_{I_{0}}^{\frac{2 p}{p-3}} L_{x}^{2 p}}+\frac{1}{2}\left\|f-f_{N}\right\|_{L_{I_{0}}^{\frac{2 p}{p-3}} L_{x}^{2 p}} .
\end{aligned}
$$

Thus, we have

$$
\left\|w_{N}\right\|_{L_{I_{0}}^{\infty} \mathcal{H}^{1}}+\left\|w_{N}\right\|_{L_{I_{0}}^{\frac{2 p}{p-3}} L_{x}^{2 p}} \leq C_{3}(T) N^{-\alpha}
$$


Therefore, by (4.18) and (4.5), there exists $N_{2}=N_{2}(T) \geq N_{1}$ such that

$$
\left\|\left(v, \partial_{t} v\right)\right\|_{L_{I_{0}}^{\infty} \mathcal{H}^{1}} \leq C_{0}(T)+T C_{3}(T) N^{-\alpha}<2 C_{0}(T)
$$

for all $N \geq N_{2}$.

This last bound (4.19) allows us to apply Lemma 4.4 again with $t_{0}=t_{*}$. And by denoting $I_{1}=\left[t_{*}, 2 t_{*}\right]$, we have

$$
t_{*}^{\frac{5-p}{2}}\left(\|v\|_{L_{I_{1}}^{p-1} L_{x}^{2 p}}^{\frac{2 p}{p-3}}+\left\|v_{N}\right\|_{L_{I_{1}}^{p-3} L_{x}^{2 p}}^{\frac{2 p}{2 p}}+\|f\|_{L_{I_{1}}^{p-1} L_{x}^{2 p}}^{p-1}+\left\|f_{N}\right\|_{L_{I_{1}}^{p-3} L_{x}^{2 p}}^{p-1}\right) \ll 1 .
$$

By the same argument as we did on $I_{0}$, there exists $N_{3}=N_{3}(T) \geq N_{2}$ such that

$$
\left\|\left(v, \partial_{t} v\right)\right\|_{L_{I_{1}}^{\infty} \mathcal{H}^{1}} \leq C_{0}(T)+T C_{3}(T)\left(C_{3}(T)+1\right) N^{-\alpha}<2 C_{0}(T)
$$

for all $N \geq N_{3}$. Notice that the bound (4.20) together with (4.19) allows us to use Lemma 4.4 again.

Iterate the above procedure, we can extend the solution $v$ onto the whole interval $[0, T]$. Moreover, there exists $N_{0}=N_{0}\left(T, t_{*}\right) \in \mathbb{N}$ such that

$$
\sup _{t \in[0, T]}\left\|\left(v, \partial_{t} v\right)\right\|_{\mathcal{H}^{1}} \leq C_{0}(T)+T\left(C_{3}(T)+1\right)^{\left[\frac{T}{t_{*}}\right]} N^{-\alpha}<2 C_{0}(T)
$$

for all $N \geq N_{0}$. Hence, we have that the solution $v$ to the equation (4.3) satisfies the energy bound (4.7) on $[0, T]$.

\section{Almost sure global well-posedness}

The following proposition can finish the proof of Theorem 1.2, see [8] and [18] for details.

Proposition 5.1 (Almost sure global well-posedness). Given $s \in\left(\frac{p-3}{p-1}, 1\right)$, for any data $\left(u_{0}, u_{1}\right) \in \mathcal{H}^{s}$, let $\left(u_{0}^{\omega}, u_{1}^{\omega}\right)$ be the randomization defined in (2.5) under the assumption (2.3). Then given any $T, \varepsilon>0$, there exists $\Omega_{T, \varepsilon} \subset \Omega$ such that

(i) $\mathbb{P}\left(\Omega_{T, \varepsilon}^{c}\right)<\varepsilon$,

(ii) for any $\omega \in \Omega_{T, \varepsilon}$, there exists a unique solution $u^{\omega}$ to the equation (1.1) with $\left.\left(u^{\omega}, \partial_{t} u^{\omega}\right)\right|_{t=0}=\left(u_{0}^{\omega}, u_{1}^{\omega}\right)$ in the class:

$$
\left(S(t)\left(u_{0}^{\omega}, u_{1}^{\omega}\right), \partial_{t} S(t)\left(u_{0}^{\omega}, u_{1}^{\omega}\right)\right)+C\left([0, T] ; \mathcal{H}^{1}\left(\mathbb{T}^{3}\right)\right) \subset C\left([0, T] ; \mathcal{H}^{s}\left(\mathbb{T}^{3}\right)\right),
$$

(iii) for any $\omega \in \Omega_{T, \varepsilon}$, the following probabilistic energy bound holds for the nonlinear part $v^{\omega}$ of the solution $u^{\omega}$ :

$$
\sup _{t \in[0, T]}\left\|\left(v^{\omega}, \partial_{t} v^{\omega}\right)\right\|_{\mathcal{H}^{1}\left(\mathbb{T}^{3}\right)}<C\left(T, \varepsilon,\left\|\left(u_{0}, u_{1}\right)\right\|_{\mathcal{H}^{s}\left(\mathbb{T}^{3}\right)}\right) .
$$


Proof. We argue in the same way as in [17]. We first construct a set $\Omega_{1}$, over which the assumption (iii) in Proposition 4.3 holds for all dyadic $N$. As usual, we denote $z^{\omega}=S(t)\left(u_{0}^{\omega}, u_{1}^{\omega}\right)$ and $z_{N}^{\omega}=P_{\leq N} S(t)\left(u_{0}^{\omega}, u_{1}^{\omega}\right)$. Taking $\alpha \in(0, s)$, we set

$$
M=M\left(T, \varepsilon,\left\|\left(u_{0}, u_{1}\right)\right\|_{\mathcal{H}^{\alpha}}\right) \sim T^{\frac{p-3}{p}}\left(\log \frac{1}{\varepsilon}\right)^{\frac{1}{2}}\left\|\left(u_{0}, u_{1}\right)\right\|_{\mathcal{H}^{\alpha}} .
$$

We then denote

$$
\Omega_{1}:=\Omega_{1}(T, \varepsilon):=\left\{\omega \in \Omega:\left\|\langle\nabla\rangle^{\alpha} z^{\omega}\right\|_{L_{T}^{\frac{2 p}{p-3}} L_{x}^{2 p}} \leq M\right\} .
$$

By Lemma 2.6(ii) we have that

$$
\mathbb{P}\left(\Omega_{1}^{c}\right)<\frac{\varepsilon}{3} .
$$

Moreover, for each $\omega \in \Omega_{1}$, we have for any $N \geq 1$

$$
\left\|z^{\omega}-z_{N}^{\omega}\right\|_{L_{T}^{\frac{2 p}{p-3}} L_{x}^{2 p}} \leq N^{-\alpha / 2}\left\|\langle\nabla\rangle^{\alpha} z^{\omega}\right\|_{L_{T}^{\frac{2 p}{p-3}} L_{x}^{2 p}} \leq M N^{-\alpha / 2} .
$$

Next, we are going to construct another subset $\Omega_{2} \subset \Omega$, over which the assumption (ii) in Proposition 4.3 holds for all dyadic $N$. Given any dyadic $N \geq 1$, apply Proposition 3.1 , we can construct $\Omega_{2}(N):=\tilde{\Omega}_{N, T, \varepsilon}$ with

$$
\mathbb{P}\left(\Omega_{2}^{c}\right)<\frac{\varepsilon}{3}
$$

such that

$$
\sup _{t \in[0, T]}\left\|\left(v_{N}^{\omega}(t), \partial_{t} v_{N}^{\omega}(t)\right)\right\|_{\mathcal{H}^{1}}<C_{0}\left(T, \varepsilon,\left\|\left(u_{0}, u_{1}\right)\right\|_{\mathcal{H}^{s}}\right)<\infty
$$

for each $\omega \in \Omega_{2}(N)$. The main point here is that the constant $C_{0}=$ $C_{0}\left(T, \varepsilon,\left\|\left(u_{0}, u_{1}\right)\right\|_{\mathcal{H}^{s}}\right)$ can be chosen to be independent of $N$.

Lastly, fix $K=\left\|\left(u_{0}, u_{1}\right)\right\|_{\mathcal{H}^{0}}$ and $2 \beta=\frac{p-3}{2 p}$ in the following. Let $t_{*}>0$ be a small number and be chosen later. By writing $[0, T]=\bigcup_{k=0}^{\left[T / t_{*}\right]} I_{k}$ with $I_{k}=\left[k t_{*},(k+1) t_{*}\right] \cap[0, T]$, define $\Omega_{3}$ by

$$
\Omega_{3}:=\bigcup_{k=0}^{\left[\frac{T}{t_{*}}\right]}\left\{\omega \in \Omega:\left\|z^{\omega}\right\|_{L_{I_{k}}^{\frac{2 p}{p-3}} L_{x}^{2 p}} \leq K\left|I_{k}\right|^{\beta}\right\} .
$$

Then by Lemma 2.6 with $\left|I_{k}\right| \leq t_{*}$, we have

$$
\mathbb{P}\left(\Omega_{3}^{c}\right) \leq \sum_{k=0}^{\left[T / t_{*}\right]} \mathbb{P}\left(\left\|z^{\omega}\right\|_{L_{I_{k}}^{\frac{2 p}{p-3} L_{x}^{2 p}}}>K\left|I_{k}\right|^{\beta}\right) \leq \frac{T}{t^{*}} \exp \left(-\frac{c}{T^{2} t_{*}^{2 \beta}}\right) .
$$

By taking $t_{*}$ even smaller if necessary, we have

$$
\mathbb{P}\left(\Omega_{3}^{c}\right) \leq \frac{T}{t_{*}} t_{*} \exp \left(-\frac{c}{2 T^{2} t_{*}^{\frac{p-3}{2 p}}}\right)=T \exp \left(-\frac{c}{2 T^{2} t_{*}^{\beta}}\right) .
$$


Hence, by choosing $t_{*}=t_{*}(T, \varepsilon)$ sufficiently small, we have

$$
\mathbb{P}\left(\Omega_{3}^{c}\right)<\frac{\varepsilon}{3}
$$

Let $\Omega_{T, \varepsilon}:=\Omega_{1} \cap \Omega_{2}\left(N_{0}\right) \cap \Omega_{3}$, where $N_{0}$ is to be chosen later. Then from (5.1), (5.3) and (5.6), we have that

$$
\mathbb{P}\left(\Omega_{T, \varepsilon}^{c}\right)<\varepsilon .
$$

By choosing $N_{0}=N_{0}\left(T, \varepsilon,\left\|\left(u_{0}, u_{1}\right)\right\|_{\mathcal{H}^{s}}\right) \gg 1$, by Proposition 4.3 , we have that there exists a solution $v^{\omega}$ to the equation $(4.3)$ on $[0, T]$ for each $\omega \in \Omega_{T, \varepsilon}$. Hence for $\omega \in \Omega_{T, \varepsilon}$, there exists a solution $u^{\omega}=z^{\omega}+v^{\omega}$ to the equation (1.1) on $[0, T]$. Moreover, there holds the estimate:

$$
\sup _{t \in[0, T]}\left\|\left(v^{\omega}(t), \partial_{t} v^{\omega}(t)\right)\right\|_{\mathcal{H}^{1}\left(\mathbb{T}^{3}\right)}<2 C_{0}\left(T, \varepsilon,\left\|\left(u_{0}, u_{1}\right)\right\|_{\mathcal{H}^{s}\left(\mathbb{T}^{3}\right)}\right)<\infty
$$

for each $\omega \in \Omega$.

Acknowledgments. We should thank Nicolas Burq for his carefully advising when we were preparing this paper, and also we should thank him and N. Tzvetkov for sharing the manuscripts on their recent works on quartic wave equation in the supercritical case. We are also grateful to G. Staffilani, who not only gave us many suggestions to correct errors in the previous versions, but also recommended us the article [21] and corrected our previous mistake on historical order of "Wiener randomization".

\section{REFERENCES}

[1] Á. Bényi, T. Oh and O. Pocovnicu, On the probabilistic Cauchy theory of the cubic nonlinear Schrödinger equation on $\mathbb{R}^{d}, d \geq 3$, Amer. Math. Soc. Transl. Ser. B 2 (2015), 1-50. MR 3350022

[2] J. Bourgain, Periodic nonlinear Schrödinger equation and invariant measures, Comm. Math. Phys. 166 1994, 1-26. MR 1309539

[3] J. Bourgain, Invariant measures for the 2D-defocusing nonlinear Schrödinger equation, Comm. Math. Phys. 176 (1996), no. 2, 421-445. MR 1374420

[4] N. Burq and G. Lebeau, Injections de Sobolev probabilistes et applications, Ann. Sci. Éc. Norm. Supér. (4) 46 (2013), no. 6, 917-962. MR 3134684

[5] N. Burq, L. Thomann and N. Tzvetkov, Global infinite energy solutions for the cubic wave equation, Bull. Soc. Math. France 143 (2015), no. 2, 301-313. MR 3351181

[6] N. Burq and N. Tzvetkov, Random data Cauchy theory for supercritical wave equations I: Local theory, Invent. Math. 173 (2007), no. 3, 449-475. MR 2425133

[7] N. Burq and N. Tzvetkov, Random data Cauchy theory for supercritical wave equations II: A global existence result, Invent. Math. 173 (2007), no. 3, 477-496. MR 2425134

[8] N. Burq and N. Tzvetkov, Probabilistic well-posedness for the cubic wave equation, J. Eur. Math. Soc. (JEMS) 16 (2014), no. 1, 1-30. MR 3141727

[9] M. Christ, J. Colliander and T. Tao, Ill-posedness for nonlinear Schrodinger and wave equations, preprint, 2003; available at arXiv:math/0311048.

[10] L. V. Kapitanski, Some generalizations of the Strichartz-Brenner inequality, Leningrad Math. J. 1 (1990), 693-726. MR 1015129

[11] M. Keel and T. Tao, Endpoint Strichartz estimates, Amer. J. Math. 120 (1998), 955980. MR 1646048 
[12] J. Lebowitz, H. Rose and E. Speer, Statistical mechanics of the nonlinear Schrödinger equation, J. Stat. Phys. 50 (1988), no. 3-4, 657-687. MR 0939505

[13] H. Lindblad and C. Sogge, On existence and scattering with minimal regularity for semilinear wave equations, J. Funct. Anal. 130 (1995), no. 2, 357-426. MR 1335386

[14] J. Lührmann and D. Mendelson, Random data Cauchy theory for nonlinear wave equations of power-type on $\mathbb{R}^{3}$, Comm. Partial Differential Equations 39 (2014), no. 12, 2262-2283. MR 3259556

[15] J. Lührmann and D. Mendelson, On the almost sure global well-posedness of energy sub-critical nonlinear wave equations on $\mathbb{R}^{3}$, New York J. Math. 22 (2016), 209-227. MR 3484682

[16] T. Oh and O. Pocovnicu, A remark on almost sure global well-posedness of the energycritical defocusing nonlinear wave equations in the periodic setting, 2015; available at arXiv: 1508.00826.

[17] T. Oh and O. Pocovnicu, Probabilistic global well-posedness of the energy-critical defocusing quintic nonlinear wave equation on $\mathbb{R}^{3}$, J. Math. Pures Appl. (9) 105 (2016), no. 3, 342-366. MR 3465807

[18] O. Pocovnicu, Almost sure global well-posedness for the energy-critical defocusing nonlinear wave equation on $\mathbb{R}^{d}, d=4,5,2014$; available at arXiv:1406.1782.

[19] T. Roy, Global analysis of the defocusing cubic wave equation in dimension 3, Ph.D. thesis, UCLA, 2008. MR 2712950

[20] B. Xia, Probabilistic well-posedness for semi-linear wave equation of power $3 \leq p<5$, 2013; available at http://www.math.u-psud.fr/ santambr/xia.pdf.

[21] T. Zhang and D. Fang, Random data Cauchy theory for the generalized incompressible Navier-Stokes equations, J. Math. Fluid Mech. 14 (2012), no. 2, 311-324. MR 2925111

Chenmin Sun, Laboratoire de Mathématiques, University Paris-Sud 11, F-91405,

\section{FRANCE}

E-mail address: chenmin.sun@u-psud.fr; csun@unice.fr

Bo Xia, Laboratoire de Mathématiques, University Paris-Sud 11, F-91405, FRANCE

E-mail address: bo.xia@math.u-psud.fr; xaboustc@gmail.com 